\title{
Deterministic Global Attitude Estimation
}

\author{
Taeyoung Lee*†, Amit Sanyal, Melvin Leok*, and N. Harris McClamroch ${ }^{\dagger}$
}

\begin{abstract}
A deterministic attitude estimation problem for a rigid body in an attitude dependent potential field with bounded measurement errors is studied. An attitude estimation scheme that does not use generalized coordinate representations of the attitude is presented here. Assuming that the initial attitude, angular velocity and measurement noise lie within given ellipsoidal bounds, an uncertainty ellipsoid that bounds the attitude and the angular velocity of the rigid body is obtained. The center of the uncertainty ellipsoid provides point estimates, and its size gives the accuracy of the estimates. The point estimates and the uncertainty ellipsoids are propagated using a Lie group variational integrator and its linearization, respectively. The estimation scheme is optimal in the sense that the attitude estimation error and the size of the uncertainty ellipsoid is minimized at each measurement instant, and it is global since the attitude is represented by a rotation matrix.
\end{abstract}

\section{INTRODUCTION}

Attitude estimation is often a prerequisite for controlling aerospace and underwater vehicles, mobile robots, and other mechanical systems moving in space. The attitude determination problem for a rigid body from vector measurements was first posed in [1]. A sample of the literature in attitude estimation can be found in [2], [3], [4].

Most existing attitude estimation schemes use generalized coordinates to represent the attitude. As is well known, minimal coordinate representations of the rotation group, like Euler angles, Rodrigues parameters, and modified Rodrigues parameters, lead to geometric or kinematic singularities. Non-minimal coordinate representations, like quaternions used in the quaternion estimation (QUEST) algorithm and its several variants ([3], [5]), have their own associated problems. Besides the extra unit norm constraint one needs to impose on the quaternion, the quaternion representation, which is diffeomorphic to $\mathrm{SU}(2)$, double covers $\mathrm{SO}(3)$. As such, it has an inevitable ambiguity in expressing the attitude.

A stochastic state estimator requires probabilistic models for the state uncertainty and the noise. However, statistical properties of the uncertainty and the noise are often not available. We usually make statistical assumptions on disturbance and noise in order to make the estimation problem

Taeyoung Lee, Aerospace Engineering, University of Michigan, Ann Arbor, MI 48109 tylee@umich. edu

Amit Sanyal, Mechanical and Aerospace Engineering, Arizona State University, Tempe, AZ 85287 sanyal@asu . edu

Melvin Leok, Mathematics, Purdue University, West Lafayette, IN 47907 mleok@math.purdue.edu

N. Harris McClamroch, Aerospace Engineering, University of Michigan, Ann Arbor, MI 48109 nhm@umich. edu

* This research has been supported in part by NSF under grant DMS0504747, and by a grant from the Rackham Graduate School, University of Michigan.

${ }^{\dagger}$ This research has been supported in part by NSF under grant ECS0244977 . mathematically tractable. In many practical situations such idealized assumptions are not appropriate, and this may cause poor estimation performance [6].

An alternative deterministic approach is to specify bounds on the uncertainty and the measurement noise without an assumption on their distribution. Noise bounds are available in many cases, and deterministic estimation is robust to the noise distribution. An efficient but flexible way to describe the bounds is using ellipsoidal sets, referred to as uncertainty ellipsoids. The idea of the deterministic estimation process is based on set theory results developed in [7]; optimal deterministic estimation problems are studied in [8] and [9] using uncertainty ellipsoids.

In this paper, we study attitude estimation problems for the uncontrolled dynamics of a rigid body in an attitudedependent potential field using uncertainty ellipsoids. The estimation scheme we present has the following important features: (1) the attitude is globally represented by a rotation matrix without using coordinates, (2) the deterministic estimator is distinguished from a Kalman or extended Kalman filter, (3) the measurement errors are assumed to be bounded but there is no restriction on their distribution, and (4) the estimates are optimal in the sense that the size of uncertainty is minimized at each estimation step.

This paper is organized as follows. The attitude determination problem from vector observations is introduced in Section II. The attitude estimation problem is formulated in Section III, and the attitude estimation scheme with angular velocity measurements is developed in Section IV. Numerical examples are presented in Section V.

\section{AtTitude DeterminAtion FROM VECTOR OBSERVATIONS}

Attitude of a rigid body is defined as the orientation of a body fixed frame with respect to a reference frame. It is represented by a rotation matrix that is a $3 \times 3$ orthogonal matrix with determinant 1 . Rotation matrices have a group structure denoted by $\mathrm{SO}(3)$. The group action of $\mathrm{SO}(3)$ on $\mathbb{R}^{3}$ transforms a vector represented in the body frame into the reference frame. In the attitude estimation problem, we measure directions in the body frame to fixed points with known directions in the reference frame. The directions in the body frame are transformed into the known reference directions by pre-multiplying by the rotation matrix defining the attitude of the rigid body. The rotation matrix can be estimated by minimizing an error between the transformed measured directions and the known reference directions.

We denote the $i$ th known direction vector in the reference frame as $e^{i} \in \mathbb{S}^{2}$, and the corresponding vector represented 
in the body frame as $b^{i} \in \mathbb{S}^{2}$. These direction vectors are normalized to have unit lengths. The $e^{i}$ and $b^{i}$ vectors are related by a rotation matrix $R \in \mathrm{SO}(3)$ that defines the attitude of the rigid body; $e^{i}=R b^{i}$, for all $i \in\{1,2, \cdots, m\}$, where $m$ is the number of measurements.

We assume that $b^{i}$ is measured by sensors in the body frame. Let the measured direction vector be $\tilde{b}^{i} \in \mathbb{S}^{2}$, which contains sensor errors, and denote the estimated rotation matrix by $\hat{R} \in \mathrm{SO}(3)$. The estimation error is given by $e^{i}-\hat{R} \tilde{b}^{i}$. The attitude determination problem consists of finding $\hat{R} \in \mathrm{SO}(3)$ such that the weighted 2 norm of those errors is minimized.

$$
\begin{gathered}
\min _{\hat{R}} \mathcal{J}=\frac{1}{2} \sum_{i=1}^{m} w_{i}\left(e^{i}-\hat{R} \tilde{b}^{i}\right)^{T}\left(e^{i}-\hat{R} \tilde{b}^{i}\right), \\
\text { subject to } \hat{R} \in \mathrm{SO}(3),
\end{gathered}
$$

where $E=\left[e^{1}, \cdots, e^{m}\right] \in \mathbb{R}^{3 \times m}, \tilde{B}=\left[\tilde{b}^{1}, \cdots, \tilde{b}^{m}\right] \in$ $\mathbb{R}^{3 \times m}$, and $W=\operatorname{diag}\left[w^{1}, \cdots, w^{m}\right] \in \mathbb{R}^{m \times m}$ is a weighting factor for each measurement.

This problem is known as Wahba's problem [1]. The original solution of Wahba's problem is given in [10], and a solution expressed in terms of quaternions (QUEST) is presented in [11]. We use the solution expressed in terms of a rotation matrix without using generalized coordinates [12]. A necessary and sufficient condition for optimality of (1) is given by

$$
\hat{R}=S L \in \mathrm{SO}(3), \quad S=S^{T}>0
$$

where $L=E W \tilde{B}^{T} \in \mathbb{R}^{3 \times 3}$ is non-singular. The unique solution of (2) is obtained by QR factorization of $L=Q_{q} Q_{r}$

$$
\hat{R}=\left(Q_{q} \sqrt{\left(Q_{r} Q_{r}^{T}\right)^{-1}} Q_{q}^{T}\right) L,
$$

where $Q_{q} \in \mathrm{SO}(3), Q_{r} \in \mathbb{R}^{3 \times 3}$ is an upper triangular matrix, and the symmetric positive definite (principal) square root is used. Equation (3) is the unique solution of Wahba's problem [12].

\section{Attitude Estimation Problem formulation}

\section{A. State bounding estimation}

We use deterministic state bounding estimation using ellipsoidal sets, referred to as uncertainty ellipsoids, to describe state uncertainty and measurement noise. This deterministic estimation procedure has steps similar to those in the Kalman filter, and is illustrated in Fig. 1. The left figure shows time evolution of an uncertainty ellipsoid, and the right figure shows a cross section at a fixed measurement instant. At the $k$ th time step, the state is bounded by an uncertainty ellipsoid centered at $\hat{x}_{k}$. This initial ellipsoid is propagated through time. Suppose that the state is measured next at the $(k+l)$ th time step, when the predicted uncertainty ellipsoid is centered at $\hat{x}_{k+l}^{f}$. At this instant, the measurement uncertainty ellipsoid is centered at $\hat{x}_{k+l}^{m}$. The actual state then lies in the intersection of the two ellipsoids. In the estimation process, we find a new ellipsoid that contains this intersection, as

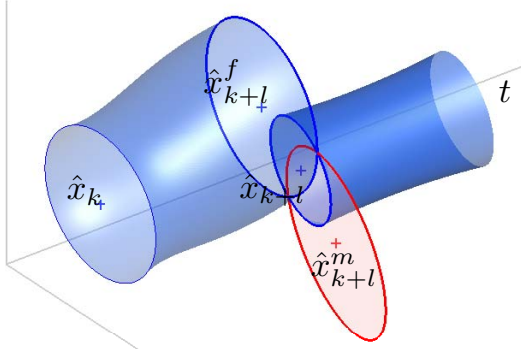

(a) Propagation of uncertainty ellipsoid

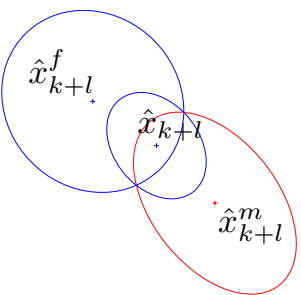

(b) Filtering procedure
Fig. 1. Uncertainty ellipsoids

shown in the right figure. The center of the new ellipsoid, $\hat{x}_{k+l}$ gives a point estimate of the state at time step $k+l$, and the magnitude of the new uncertainty ellipsoid measures the estimation accuracy. The deterministic estimates are optimal in the sense that the sizes of the ellipsoids are minimized.

\section{B. Equations of motion}

We consider estimation of the attitude dynamics of a rigid body in the presence of an attitude dependent potential, $U(\cdot): \mathrm{SO}(3) \mapsto \mathbb{R}, R \in \mathrm{SO}(3)$. Systems that can be so modeled include a free rigid body, spacecraft on a circular orbit with gravity gradient effects [13], or a 3D pendulum [14]. The continuous equations of motion are

$$
\begin{gathered}
J \dot{\Omega}+\Omega \times J \Omega=M, \\
\dot{R}=R S(\Omega),
\end{gathered}
$$

where $J \in \mathbb{R}^{3 \times 3}$ is the moment of inertia matrix of the rigid body, $\Omega \in \mathbb{R}^{3}$ is the angular velocity of the body expressed in the body fixed frame, and $S(\cdot): \mathbb{R}^{3} \mapsto \mathfrak{s o}(3)$ is a skew mapping defined by $S(x) y=x \times y$ for all $x, y \in \mathbb{R}^{3}$. The vector $M \in \mathbb{R}^{3}$ is the moment due to the potential, determined by $S(M)=\frac{\partial U}{\partial R} R-R^{T} \frac{\partial U}{\partial R}$, or more explicitly,

$$
M=r_{1} \times v_{r_{1}}+r_{2} \times v_{r_{2}}+r_{3} \times v_{r_{3}},
$$

where $r_{i}, v_{r_{i}} \in \mathbb{R}^{1 \times 3}$ are the $i$ th row vectors of $R$ and $\frac{\partial U}{\partial R}$, respectively.

General numerical integration methods like the popular Runge-Kutta schemes, typically preserve neither first integrals nor the characteristics of the configuration space, $\mathrm{SO}(3)$. In particular, the orthogonal structure of the rotation matrices is not preserved numerically. It is often proposed to parameterize (5) by Euler angles or quaternions instead of integrating (5) directly. However, Euler angles yield only local representations of the attitude and they have singularities. Unit quaternions do not exhibit singularities, but they have the manifold structure of the three sphere $\mathbb{S}^{3}$, and double cover $\mathrm{SO}(3)$. Consequently, the unit quaternion representing the attitude is inevitably ambiguous. In addition, general numerical integration methods do not preserve the unit length constraint. Therefore, quaternions have the same numerical drift problem as rotation matrices. 
Lie group variational integrators preserve the group structure without the use of local charts, reprojection, or constraints, they are symplectic and momentum preserving, and they exhibit good energy behavior for an exponentially long time period. The following Lie group variational integrator for the attitude dynamics of a rigid body is presented in [14]:

$$
\begin{gathered}
h S\left(J \Omega_{k}+\frac{h}{2} M_{k}\right)=F_{k} J_{d}-J_{d} F_{k}^{T}, \\
R_{k+1}=R_{k} F_{k}, \\
J \Omega_{k+1}=F_{k}^{T} J \Omega_{k}+\frac{h}{2} F_{k}^{T} M_{k}+\frac{h}{2} M_{k+1},
\end{gathered}
$$

where $J_{d} \in \mathbb{R}^{3 \times 3}$ is a nonstandard moment of inertia matrix defined by $J_{d}=\frac{1}{2} \operatorname{tr}[J] I_{3 \times 3}-J$, and $F_{k} \in \mathrm{SO}(3)$ is the relative attitude over an integration step. The constant $h \in \mathbb{R}$ is the integration step size, and the subscript $k$ denotes the $k$ th integration step. This integrator yields a map $\left(R_{k}, \Omega_{k}\right) \mapsto$ $\left(R_{k+1}, \Omega_{k+1}\right)$ by solving (7) to obtain $F_{k} \in \mathrm{SO}(3)$ and substituting it into (8) and (9) to obtain $R_{k+1}$ and $\Omega_{k+1}$.

It preserves the orthogonal structure of $\mathrm{SO}(3)$ because the rotation matrix is updated by a product of two rotation matrices in (8). Since this integrator is obtained from a discrete variational principle, it is symplectic, momentum preserving, and has good energy behavior, properties that are characteristic of variational integrators.

\section{Uncertainty Ellipsoid}

An uncertainty ellipsoid in $\mathbb{R}^{n}$ is defined as

$$
\mathcal{E}_{\mathbb{R}^{n}}(\hat{x}, P)=\left\{x \in \mathbb{R}^{n} \mid(x-\hat{x})^{T} P^{-1}(x-\hat{x}) \leq 1\right\},
$$

where $\hat{x} \in \mathbb{R}^{n}$, and $P \in \mathbb{R}^{n \times n}$ is a symmetric positive definite matrix. We call $\hat{x}$ the center of the uncertainty ellipsoid, and $P$ is the uncertainty matrix that determines the size and the shape of the uncertainty ellipsoid. The size of an uncertainty ellipsoid is measured by $\operatorname{tr}[P]$ which is the sum of the squares of the semi principal axes of the ellipsoid.

The state evolves in the 6 dimensional tangent bundle, $\operatorname{TSO}(3)$. We identify $\operatorname{TSO}(3)$ with $\mathrm{SO}(3) \times \mathfrak{s o}(3)$ by left trivialization, and we identify $\mathfrak{s o}(3)$ with $\mathbb{R}^{3}$ by the isomorphism $S(\cdot)$. The uncertainty ellipsoid centered at $(\hat{R}, \hat{\Omega}) \in$ $\operatorname{TSO}(3)$ is induced from an uncertainty ellipsoid in $\mathbb{R}^{6}$;

$\mathcal{E}(\hat{R}, \hat{\Omega}, P)=\left\{R \in \mathrm{SO}(3), \Omega \in \mathbb{R}^{3} \mid\left[\begin{array}{c}\zeta \\ \delta \Omega\end{array}\right] \in \mathcal{E}_{\mathbb{R}^{6}}\left(0_{6}, P\right)\right\}$

where $S(\zeta)=\operatorname{logm}\left(\hat{R}^{T} R\right) \in \mathfrak{s o}(3), \delta \Omega=\Omega-\hat{\Omega} \in \mathbb{R}^{3}$, and $P \in \mathbb{R}^{6 \times 6}$ is a symmetric positive definite matrix. An element $(R, \Omega) \in \mathcal{E}(\hat{R}, \hat{\Omega}, P)$ can be written as

$$
R=\hat{R} e^{S(\zeta)}, \quad \Omega=\hat{\Omega}+\delta \Omega,
$$

for some $x=[\zeta ; \delta \Omega] \in \mathbb{R}^{6}$ satisfying $x^{T} P^{-1} x \leq 1$.

\section{Uncertainty model}

We define the measurement error models for the direction vector and for the angular velocity. The measurement error is modeled by rotation of the measured direction;

$$
\begin{aligned}
b^{i} & =e^{S\left(\nu^{i}\right)} \tilde{b}^{i}, \\
& \simeq \tilde{b}^{i}+S\left(\nu^{i}\right) \tilde{b}^{i},
\end{aligned}
$$

where $\nu^{i} \in \mathbb{R}^{3}$ is the sensor error, which represents the Euler axis of rotation vector from $\tilde{b}^{i}$ to $b^{i}$, and $\left\|\nu^{i}\right\|$ is the corresponding rotation angle in radians. The approximation is obtained by assuming that the measurement error is small.

The angular velocity measurement errors are modeled as

$$
\Omega_{k}=\tilde{\Omega}_{k}+v_{k},
$$

where $\tilde{\Omega}_{k} \in \mathbb{R}^{3}$ is the measured angular velocity, and $v_{k} \in$ $\mathbb{R}^{3}$ is an additive error.

We assume that the initial conditions and the sensor noise are bounded by prescribed uncertainty ellipsoids.

$$
\begin{gathered}
\left(R_{0}, \Omega_{0}\right) \in \mathcal{E}\left(\hat{R}_{0}, \hat{\Omega}_{0}, P_{0}\right), \\
\nu_{k}^{i} \in \mathcal{E}_{\mathbb{R}^{3}}\left(0, S_{k}^{i}\right), \\
v_{k} \in \mathcal{E}_{\mathbb{R}^{3}}\left(0, T_{k}\right),
\end{gathered}
$$

where $P_{0} \in \mathbb{R}^{6 \times 6}, S_{k}^{i}, T_{k} \in \mathbb{R}^{3 \times 3}$ are symmetric positive definite matrices that define the shape and the size of the uncertainty ellipsoids.

\section{Attitude Estimation with AtTitude AND Angular Velocity Measurements}

In this section, we develop a deterministic estimator for the attitude and the angular velocity of a rigid body assuming that both attitude and angular velocity measurements are available. The estimator consists of three stages; flow update, measurement update, and filtering. The flow update predicts the uncertainty ellipsoid in the future. The measurement update obtains an uncertainty ellipsoid using new measurements and the sensor error model. Filtering obtains a new uncertainty ellipsoid compatible with the predicted and the measured uncertainty ellipsoids.

The subscript $k$ denotes the $k$ th discrete index, and the superscript $i$ denotes $i$ th directional sensor. The superscripts $f$ and $m$ denote the variables related to the flow update and the measurement update, respectively. $\tilde{\text { denotes a measured }}$ variable, and $\hat{\imath}$ denotes an estimated variable.

\section{A. Flow update}

Suppose that the attitude and the angular momentum at the $k$ th step lie in a given uncertainty ellipsoid

$$
\left(R_{k}, \Omega_{k}\right) \in \mathcal{E}\left(\hat{R}_{k}, \hat{\Omega}_{k}, P_{k}\right)
$$

and that new measurements are taken at $(k+l)$ th time step. Flow update predicts the center and the uncertainty matrix that define the uncertainty ellipsoid at the $(k+l)$ th step using the given uncertainty ellipsoid at the $k$ th step. Since the attitude dynamics is nonlinear, the admissible boundary of the state at the $(k+l)$ th step is not an ellipsoid in general. 
We assume that the uncertainty ellipsoid at the $k$ th step is sufficiently small that states in the uncertainty ellipsoid can be approximated using the linearized equations of motion.

Center: For the given center $\left(\hat{R}_{k}, \hat{\Omega}_{k}\right)$, the center of the uncertainty ellipsoid at step $(k+l)$ is $\left(\hat{R}_{k+l}^{f}, \hat{\Omega}_{k+l}^{f}\right)$ obtained using the discrete equations of motion, (7), (8), and (9):

$$
\begin{gathered}
h S\left(J \hat{\Omega}_{k}+\frac{h}{2} \hat{M}_{k}\right)=\hat{F}_{k} J_{d}-J_{d} \hat{F}_{k}^{T}, \\
\hat{R}_{k+1}^{f}=\hat{R}_{k} \hat{F}_{k}, \\
J \hat{\Omega}_{k+1}^{f}=\hat{F}_{k}^{T} \hat{\Omega}_{k}+\frac{h}{2} \hat{F}_{k}^{T} \hat{M}_{k}+\frac{h}{2} \hat{M}_{k+1} .
\end{gathered}
$$

This integrator yields a map $\left(\hat{R}_{k}, \hat{\Omega}_{k}\right) \mapsto\left(\hat{R}_{k+1}^{f}, \hat{\Omega}_{k+1}^{f}\right)$, and this process is repeatedly applied to find the center at the $(k+l)$ th step, $\left(\hat{R}_{k+l}^{f}, \hat{\Omega}_{k+l}^{f}\right)$.

Uncertainty matrix: At the $(k+1)$ th step, the state is represented by perturbations from the center $\left(\hat{R}_{k+1}^{f}, \hat{\Omega}_{k+1}^{f}\right)$ :

$$
\begin{aligned}
& R_{k+1}=\hat{R}_{k+1}^{f} e^{S\left(\zeta_{k+1}^{f}\right)}, \\
& \Omega_{k+1}=\hat{\Omega}_{k+1}^{f}+\delta \Omega_{k+1}^{f},
\end{aligned}
$$

for some $\zeta_{k+1}^{f}, \delta \Omega_{k+1}^{f} \in \mathbb{R}^{3}$. The uncertainty matrix at the $(k+1)$ th step is obtained by finding a bound on $\zeta_{k+1}^{f}, \delta \Omega_{k+1}^{f} \in \mathbb{R}^{3}$. Assume that the uncertainty ellipsoid at the $k$ th step is sufficiently small. Then, $\zeta_{k+1}^{f}, \delta \Omega_{k+1}^{f}$ are represented by the following linear equations in [13]

$$
x_{k+1}^{f}=A_{k}^{f} x_{k},
$$

where $x_{k}=\left[\zeta_{k} ; \delta \Omega_{k}\right] \in \mathbb{R}^{6}$, and $A_{k}^{f} \in \mathbb{R}^{6 \times 6}$ can be suitably defined. Since $\left(R_{k}, \Omega_{k}\right) \in \mathcal{E}\left(\hat{R}_{k}, \hat{\Omega}_{k}, P_{k}\right), x_{k} \in \mathcal{E}_{\mathbb{R}^{6}}\left(0, P_{k}\right)$ by the definition of the uncertainty ellipsoid given in (11). Then we can show that $A_{k}^{f} x_{k}$ lies in

$$
A_{k}^{f} x_{k} \in \mathcal{E}_{\mathbb{R}^{6}}\left(0, A_{k}^{f} P_{k}\left(A_{k}^{f}\right)^{T}\right) .
$$

Thus, the uncertainty matrix at the $(k+1)$ th step is given by

$$
P_{k+1}^{f}=A_{k}^{f} P_{k}\left(A_{k}^{f}\right)^{T}
$$

In summary, the uncertainty ellipsoid at the $(k+l)$ th step is computed using (17), (18), (19), and (20) as:

$$
\left(R_{k+l}, \Omega_{k+l}\right) \in \mathcal{E}\left(\hat{R}_{k+l}^{f}, \hat{\Omega}_{k+l}^{f}, P_{k+l}^{f}\right) .
$$

\section{B. Measurement update}

The measurement update finds an uncertainty ellipsoid in the state space using the measurements and sensor error models. The measured attitude and the angular velocity define the center of the measurement uncertainty ellipsoid, and the sensor error models give the uncertainty matrix.

Center: The center of the uncertainty ellipsoid, $\left(\hat{R}_{k+l}^{m}, \hat{\Omega}_{k+l}^{m}\right)$ is obtained from measurements. Let the measured directions to the known points be $\tilde{B}_{k+l}=\left[\tilde{b}^{1}, \cdots, \tilde{b}^{m}\right] \in \mathbb{R}^{3 \times m}$. Then, the attitude $\hat{R}_{k+l}^{m}$ satisfies the following necessary condition given in (2)

$$
\left(\hat{R}_{k+l}^{m}\right)^{T} \tilde{L}_{k+l}-\tilde{L}_{k+l}^{T} \hat{R}_{k+l}^{m}=0
$$

where $\tilde{L}_{k+l}=E_{k+l} W_{k+l} \tilde{B}_{k+l}^{T} \in \mathbb{R}^{3 \times 3}$. The attitude matrix is given by a $\mathrm{QR}$ factorization of $\tilde{L}_{k+l}$ as in (3)

$$
\hat{R}_{k+l}^{m}=\left(Q_{q} \sqrt{\left(Q_{r} Q_{r}^{T}\right)^{-1}} Q_{q}^{T}\right) \tilde{L}_{k+l},
$$

where $Q_{q} \in \mathrm{SO}(3)$ is an orthogonal matrix and $Q_{r} \in \mathbb{R}^{3 \times 3}$ is a upper triangular matrix satisfying $\tilde{L}_{k+l}=Q_{q} Q_{r}$.

The angular velocity is measured directly by

$$
\hat{\Omega}_{k+l}^{m}=\tilde{\Omega}_{k+l} .
$$

Uncertainty matrix: We represent the actual state at the $(k+l)$ th step as perturbations from the measured center:

$$
\begin{aligned}
& R_{k+l}=\hat{R}_{k+l}^{m} e^{S\left(\zeta_{k+l}^{m}\right)}, \\
& \Omega_{k+l}=\hat{\Omega}_{k+l}^{m}+\delta \Omega_{k+l}^{m},
\end{aligned}
$$

for $\zeta_{k+l}^{m}, \delta \Omega_{k+l}^{m} \in \mathbb{R}^{3}$. The uncertainty matrix is obtained by finding a bound on $\zeta_{k+l}^{m}, \delta \Omega_{k+l}^{m}$.

We transform the uncertainties in measuring the body directions to known fixed points into uncertainties in the rotation matrix by (22). Using the error model in (12), the actual directions corresponding to $B_{k+l}$ are given by

$$
B_{k+l}=\tilde{B}_{k+l}+\delta \tilde{B}_{k+l}
$$

where $\delta B_{k+l}=\left[S\left(\nu^{1}\right) \tilde{b}^{1}, \cdots, S\left(\nu^{m}\right) \tilde{b}^{m}\right] \in \mathbb{R}^{3 \times m}$.

The actual directions $B_{k+l}$ and the actual attitude $R_{k+l}$ at the $(k+l)$ th step also satisfy $(23)$;

$$
R_{k+l}^{T} L_{k+l}-L_{k+l}^{T} R_{k+l}=0,
$$

where $L_{k+l}=E_{k+l} W_{k+l} B_{k+l}^{T} \in \mathbb{R}^{3 \times 3}$. Substitute (25) and (27) into (28), and use $S(x) A+A^{T} S(x)=$ $S\left(\left\{\operatorname{tr}[A] I_{3 \times 3}-A\right\} x\right)$ for $A \in \mathbb{R}^{3 \times 3}, x \in \mathbb{R}^{3}$, to get:

$$
\begin{aligned}
& \left\{\operatorname{tr}\left[\left(\hat{R}_{k+l}^{m}\right)^{T} \tilde{L}_{k+l}\right]-\left(\hat{R}_{k+l}^{m}\right)^{T} \tilde{L}_{k+l}\right\} \zeta_{k+l}^{m} \\
& =-\sum_{i=1}^{m} w_{i}\left\{\operatorname{tr}\left[\tilde{b}^{i}\left(e^{i}\right)^{T} \hat{R}_{k+l}^{m}\right] I_{3 \times 3}-\tilde{b}^{i}\left(e^{i}\right)^{T} \hat{R}_{k+l}^{m}\right\} \nu^{i} .
\end{aligned}
$$

We can rewrite the above equation as

$$
\zeta_{k+l}^{m}=\sum_{i=1}^{m} \mathcal{A}_{k+l}^{m, i} \nu^{i}
$$

where $\mathcal{A}_{k+l}^{m, i} \in \mathbb{R}^{3 \times 3}$ is defined appropriately.

The perturbation of the angular velocity $\delta \Omega_{k+l}^{m}$ is equal to the angular velocity measurement error $v_{k+l}$,

$$
\delta \Omega_{k+l}^{m}=v_{k+l} .
$$

Define $x_{k+l}^{m}=\left[\zeta_{k+l}^{m} ; \delta \Omega_{k+l}^{m}\right] \in \mathbb{R}^{6}$. Using (29) and (30),

$$
x_{k+l}^{m}=H_{1} \sum_{i=1}^{m} \mathcal{A}_{k+l}^{m, i} \nu_{k+l}^{i}+H_{2} v_{k+l},
$$

where $H_{1}=\left[I_{3 \times 3}, 0_{3 \times 3}\right]^{T}, H_{2}=\left[0_{3 \times 3}, I_{3 \times 3}\right]^{T} \in \mathbb{R}^{6 \times 3}$. This expresses $x_{k+l}^{m}$ as a linear combination of the sensor errors $\nu^{i}$ and $v$. Using the measurement uncertainties (15) 
and (16), we can show that the terms in the right hand side of the above equation are in the following uncertainty ellipsoids:

$$
\begin{aligned}
H_{1} \mathcal{A}_{k+l}^{m, i} \nu_{k+l}^{i} & \in \mathcal{E}_{\mathbb{R}^{6}}\left(0, H_{1} \mathcal{A}_{k+l}^{m, i} S_{k+l}^{i}\left(\mathcal{A}_{k+l}^{m, i}\right)^{T} H_{1}^{T}\right), \\
H_{2} v_{k+l} & \in \mathcal{E}_{\mathbb{R}^{6}}\left(0, H_{2} T_{k+l} H_{2}^{T}\right) .
\end{aligned}
$$

Thus, the uncertainty ellipsoid for $x_{k+l}^{m}$ is obtained as the vector sum of the above uncertainty ellipsoids. The measurement update obtains a minimal ellipsoid that contains the vector sum of these uncertainty ellipsoids. Using expressions for such a minimal ellipsoid given in [8] and [9], we get:

$$
\begin{aligned}
P_{k+l}^{m}= & \left\{\sum_{i=1}^{m} \sqrt{\operatorname{tr}\left[P_{k+l, R}^{m, i}\right]}+\sqrt{\operatorname{tr}\left[P_{k+l, \Omega}^{m}\right]}\right\} \\
& \times\left\{\sum_{i=1}^{m} \frac{P_{k+l, R}^{m, i}}{\sqrt{\operatorname{tr}\left[P_{k+l, R}^{m, i}\right]}}+\frac{P_{k+l, \Omega}^{m}}{\sqrt{\operatorname{tr}\left[P_{k+l, \Omega}^{m}\right]}}\right\},
\end{aligned}
$$

where

$$
\begin{aligned}
& P_{k+l, R}^{m, i}=H_{1} \mathcal{A}_{k+l}^{m, i} S_{k+l}^{i}\left(\mathcal{A}_{k+l}^{m, i}\right)^{T} H_{1}^{T}, \\
& P_{k+l, \Omega}^{m}=H_{2} T_{k+l} H_{2}^{T} .
\end{aligned}
$$

In summary, the measured uncertainty ellipsoid at the $(k+$ l)th step is defined by (23), (24), and (31);

$$
\left(R_{k+l}, \Omega_{k+l}\right) \in \mathcal{E}\left(\hat{R}_{k+l}^{m}, \hat{\Omega}_{k+l}^{m}, P_{k+l}^{m}\right) .
$$

\section{Filtering procedure}

The filtering procedure obtains a new uncertainty ellipsoid compatible with both the predicted and the measured uncertainty ellipsoids. From (21) and (32), we know that:

$$
\left(R_{k+l}, \Omega_{k+l}\right) \in \mathcal{E}\left(\hat{R}_{k+l}^{f}, \hat{\Omega}_{k+l}^{f}, P_{k+l}^{f}\right) \bigcap \mathcal{E}\left(\hat{R}_{k+l}^{m}, \hat{\Omega}_{k+l}^{m}, P_{k+l}^{m}\right) .
$$

The intersection of two ellipsoids is not generally an ellipsoid, and it is inefficient to describe an irregular subset in the multidimensional space numerically. We find a minimal uncertainty ellipsoid containing this intersection. We omit the subscript $(k+l)$ here for convenience.

The measurement uncertainty ellipsoid, $\mathcal{E}\left(\hat{R}^{m}, \hat{\Omega}^{m}, P^{m}\right)$, is identified by its center $\left(\hat{R}^{m}, \hat{\Omega}^{m}\right)$, and the uncertainty ellipsoid in $\mathbb{R}^{6}$ :

$$
\left(\zeta^{m}, \delta \Omega^{m}\right) \in \mathcal{E}_{\mathbb{R}^{6}}\left(0_{6 \times 1}, P^{m}\right),
$$

where $S\left(\zeta^{m}\right)=\operatorname{logm}\left(\left(\hat{R}^{m}\right)^{T} R\right) \in \mathfrak{s o}(3), \delta \Omega^{m}=$ $\Omega-\hat{\Omega}^{m} \in \mathbb{R}^{3}$. Similarly, the flow uncertainty ellipsoid, $\mathcal{E}\left(\hat{R}^{f}, \hat{\Omega}^{f}, P^{f}\right)$, is identified by its center $\left(\hat{R}^{f}, \hat{\Omega}^{f}\right)$, and the uncertainty ellipsoid in $\mathbb{R}^{6}$ :

$$
\left(\zeta^{f}, \delta \Omega^{f}\right) \in \mathcal{E}_{\mathbb{R}^{6}}\left(0_{6 \times 1}, P^{f}\right),
$$

where $S\left(\zeta^{f}\right)=\operatorname{logm}\left(\left(\hat{R}^{f}\right)^{T} R\right) \in \mathfrak{s o}(3), \delta \Omega^{f}=\Omega-\hat{\Omega}^{f} \in$ $\mathbb{R}^{3}$. An element $\left(R^{f}, \Omega^{f}\right) \in \mathcal{E}\left(\hat{R}^{f}, \hat{\Omega}^{f}, P^{f}\right)$ is given by

$$
\begin{aligned}
& R^{f}=\hat{R}^{f} e^{S\left(\zeta^{f}\right)}, \\
& \Omega^{f}=\hat{\Omega}^{f}+\delta \Omega^{f} .
\end{aligned}
$$

Define $\hat{\zeta}^{m f}, \delta \hat{\Omega}^{m f} \in \mathbb{R}^{3}$ such that

$$
\begin{aligned}
& \hat{R}^{f}=\hat{R}^{m} e^{S\left(\hat{\zeta}^{m f}\right)}, \\
& \hat{\Omega}^{f}=\hat{\Omega}^{m}+\delta \hat{\Omega}^{m f} .
\end{aligned}
$$

Thus, $\hat{\zeta}^{m f}, \delta \hat{\Omega}^{m f}$ represent the difference between the centers of the two ellipsoids.

Substituting (37), (38) into (35), (36), we obtain

$$
\begin{aligned}
R^{f} & =\hat{R}^{m} e^{S\left(\hat{\zeta}^{m f}\right)} e^{S\left(\zeta^{f}\right)}, \\
& \simeq \hat{R}^{m} e^{S\left(\hat{\zeta}^{m f}+\zeta^{f}\right)}, \\
\Omega^{f} & =\hat{\Omega}^{m}+\left(\delta \hat{\Omega}^{m f}+\delta \Omega^{f}\right),
\end{aligned}
$$

where we assumed that $\hat{\zeta}^{m f}, \zeta^{f}$ are sufficiently small. Thus, the uncertainty ellipsoid obtained by the flow update, $\mathcal{E}\left(\hat{R}^{f}, \hat{\Omega}^{f}, P^{f}\right)$ is identified by the measured $\left(\hat{R}^{m}, \hat{\Omega}^{m}\right)$ and the following uncertainty ellipsoid in $\mathbb{R}^{6}$ :

$$
\mathcal{E}_{\mathbb{R}^{6}}\left(\hat{x}^{m f}, P^{f}\right),
$$

where $\hat{x}^{m f}=\left[\hat{\zeta}^{m f} ; \delta \hat{\Omega}^{m f}\right] \in \mathbb{R}^{6}$.

We seek a minimal ellipsoid that contains the intersection:

$$
\mathcal{E}_{\mathbb{R}^{6}}\left(0_{6 \times 1}, P^{m}\right) \bigcap \mathcal{E}_{\mathbb{R}^{6}}\left(\hat{x}^{m f}, P^{f}\right) \subset \mathcal{E}_{\mathbb{R}^{6}}(\hat{x}, P),
$$

where $\hat{x}=[\hat{\zeta} ; \delta \hat{\Omega}] \in \mathbb{R}^{6}$. Using the expression for a minimal ellipsoid containing the intersection of two ellipsoids presented in [8], $\hat{x}$ and $P$ are given by

$$
\begin{aligned}
& \hat{x}=L \hat{x}^{m f}, \\
& P=\beta(q)(I-L) P^{m},
\end{aligned}
$$

where

$$
\begin{aligned}
\beta(q) & =1+q-\left(\hat{x}^{m f}\right)^{T}\left(P^{m}\right)^{-1} L \hat{x}^{m f}, \\
L & =P^{m}\left(P^{m}+q^{-1} P^{f}\right)^{-1} .
\end{aligned}
$$

The constant $q$ is chosen to minimize $\operatorname{tr}[P]$. We convert $\hat{x}$ to points in $\operatorname{TSO}(3)$ using the common center $\left(\hat{R}^{m}, \hat{\Omega}^{m}\right)$.

In summary, the uncertainty ellipsoid at $(k+l)$ th step is

$$
\left(R_{k+l}, \Omega_{k+l}\right) \in \mathcal{E}\left(\hat{R}_{k+l}, \hat{\Omega}_{k+l}, P_{k+l}\right),
$$

where

$$
\hat{R}_{k+l}=\hat{R}_{k+l}^{m} e^{S(\hat{\zeta})}, \quad \hat{\Omega}_{k+l}=\hat{\Omega}_{k+l}^{m}+\delta \hat{\Omega}, \quad P_{k+l}=P .
$$

\section{Properties of the estimator}

The steps outlined above are repeated to get a dynamic filter. This attitude estimator has no singularities since the attitude is represented by a rotation matrix. Orthogonality of the rotation matrix is preserved as it is updated by the structure-preserving Lie group variational integrator. This estimator can be used for highly nonlinear large angle maneuvers of a rigid body. It is also robust to the distribution of the sensor noise since we only use ellipsoidal bounds on the noise. The measurements need not be periodic, the estimation is repeated whenever new measurements become available. We can also extend this attitude estimator to the case when angular velocity measurements are not available. The filtering step is modified to find an intersection of the non-degenerate predicted uncertainty ellipsoid and the degenerate measurement uncertainty ellipsoid. 


\section{NUmerical Simulation}

Numerical simulation results are presented for estimation of the attitude dynamics of an uncontrolled rigid spacecraft in a circular orbit about a large central body, including gravity gradient effects. The on orbit spacecraft model is given in [13].

The mass, length and time dimensions are normalized by the spacecraft mass, the maximum length of the spacecraft, and the orbital angular velocity, respectively. The inertia of the spacecraft is chosen as $J=\operatorname{diag}[1,2.8,2]$. The maneuver is an arbitrary large attitude change completed in a quarter of the orbit. The initial conditions are chosen as

$R_{0}=\operatorname{diag}[-1,-1,1], \quad \Omega_{0}=[2.316,0.446,-0.591] \mathrm{rad} / \mathrm{s}$, $\hat{R}_{0}=I_{3 \times 3}, \quad \hat{\Omega}_{0}=[2.116,0.546,-0.891] \mathrm{rad} / \mathrm{s}$.

The corresponding initial estimation errors are $\left\|\zeta_{0}\right\|=$ $180 \mathrm{deg},\left\|\delta \Omega_{0}\right\|=21.43 \frac{\pi}{180} \mathrm{rad} / \mathrm{s}$. Note that the actual initial attitude is opposite to the estimated initial attitude. The initial uncertainty matrix is given by

$$
P_{0}=2 \operatorname{diag}\left[\pi^{2}[1,1,1],\left(\frac{\pi}{6}\right)^{2}[1,1,1]\right],
$$

so that $x_{0}^{T} P_{0}^{-1} x_{0}=0.7553 \leq 1$.

We assume that the measurements are available ten times per quarter orbit. The measurement noise is assumed to be normally distributed with uncertainty matrices given by

$$
S_{k}^{i}=\left(7 \frac{\pi}{180}\right)^{2} I_{3 \times 3} \mathrm{rad}^{2}, \quad T_{k}=\left(7 \frac{\pi}{180}\right)^{2} I_{3 \times 3} \operatorname{rad}^{2} / \mathrm{s}^{2} .
$$

We consider two cases. Fig. 2 shows simulation results when both the attitude and the angular velocity are measured. Fig. 3 shows simulation results when angular velocity measurements are not available. In each figure, the left plot shows the attitude and angular velocity estimation errors, and the right plot shows the size of the uncertainty ellipsoid. The estimation errors and the size of uncertainty decrease rapidly after the first measurement. When the angular velocity measurements are not available, the estimation error for the angular velocity converges relatively slowly as seen in Fig. 3.(a). For both cases, the terminal attitude error, and the terminal angular velocity error are less than $0.88 \mathrm{deg}$, and $0.04 \mathrm{rad} / \mathrm{s}$, respectively.

\section{CONCLUSION}

A deterministic estimator for the attitude dynamics of a rigid body in a potential field with bounded measurement errors is presented. An uncertainty ellipsoid is obtained at each estimation step, and the dynamics is propagated using Lie group variational integrators. The center of the uncertainty ellipsoid is the point estimate, and its size determines the accuracy of the estimate. The estimation scheme is optimal in the sense that the size of the uncertainty is minimized at each estimation step. It is also global and robust to the distribution of measurement noise. This estimator can be extended to include the effects of process noise and to the case when only attitude measurements are available. These extensions are not described in this paper.
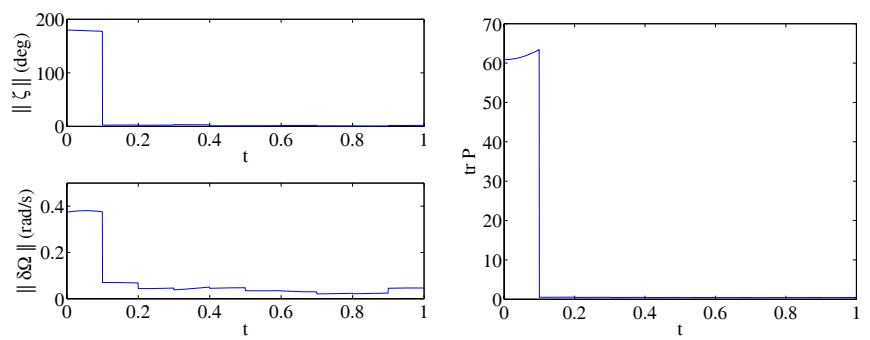

(a) Estimation error $\left\|\zeta_{k}\right\|,\left\|\delta \Omega_{k}\right\|$

(b) Size of uncertainty $\operatorname{tr}\left[P_{k}\right]$

Fig. 2. Estimation with attitude and angular velocity measurement
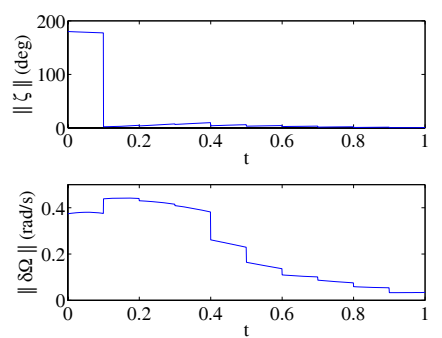

(a) Estimation error $\left\|\zeta_{k}\right\|,\left\|\delta \Omega_{k}\right\|$

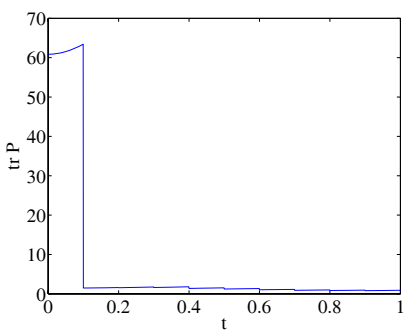

(b) Size of uncertainty $\operatorname{tr}\left[P_{k}\right]$

Fig. 3. Estimation with attitude measurement only

\section{REFERENCES}

[1] G. Wahba, "A least squares estimate of satellite attitude, Problem 651," SIAM Review, vol. 7, no. 5, p. 409, 1965.

[2] I. Y. Bar-Itzhack and Y. Oshman, "Attitude determination from vector observations; quaternion estimation," IEEE Transactions on Aerospace and Electronic Systems, vol. 21, no. 1, pp. 128-136, 1985.

[3] M. D. Shuster, "Kalman filtering of spacecraft attitude and the QUEST model," Journal of the Astronautical Sciences, vol. 38, no. 3, pp. 377393, 1990.

[4] H. Rehbinder and X. Hu, "Drift-free attitude estimation for accelerated rigid bodies," Automatica, vol. 40, no. 4, pp. 653-659, 2004.

[5] M. L. Psiaki, "Attitude determination filtering via extended quaternion estimation," AIAA Journal of Guidance, Control and Dynamics, vol. 23, no. 2, pp. 206-214, 2000.

[6] Y. Theodor, U. Shaked, and C. E. de Souza, "A game theory approach to robust discrete-time $H_{\infty}$-estimation," IEEE Transactions on Signal Processing, vol. 42, no. 6, pp. 1486-1495, 1994.

[7] F. C. Schweppe, "Recursive state estimation: Unknown but bounded errors and system inputs," IEEE Transactions on Automatic Control, vol. 13, no. 1, pp. 22-28, 1968.

[8] D. G. Maksarov and J. P. Norton, "State bounding with ellipsoidal set description of the uncertainty," International Journal of Control, vol. 65, no. 5, pp. 847-866, 1996.

[9] C. Durieu, E. Walter, and B. Polyak, "Multi-input multi-output ellipsoidal state bounding," Journal of Optimization Theory and Applications, vol. 111, no. 2, pp. 273-303, 2001.

[10] J. L. Farrell, J. C. Stuelpnagel, R. H. Wessner, J. R. Velman, and J. E. Brock, "A least squares estimate of satellite attitude, Solution 65-1," SIAM Review, vol. 8, no. 3, pp. 384-386, 1966.

[11] M. D. Shuster and S. D. Oh, "Three-axis attitude determination from vector observations," Journal of Guidance Control and Dynamics, vol. 4 , no. 1 , pp. 70-77, 1981.

[12] A. K. Sanyal, "Optimal attitude estimation and filtering without using local coordinates, Part I: Uncontrolled and deterministic attitude dynamics," in Proceedings of the American Control Conference, 2006, pp. 5734-5739.

[13] T. Lee, M. Leok, and N. H. McClamroch, "Attitude maneuvers of a rigid spacecraft in a circular orbit," in Proceedings of the American Control Conference, 2006, pp. 1742-1747.

[14] _ _ "A Lie group variational integrator for the attitude dynamics of a rigid body with applications to the 3D pendulum," in Proceedings of the IEEE Conference on Control Applications, 2005, pp. 962-967. 